\title{
2131. Nonlinear factor analysis and its application to acoustical source separation and identification
}

\author{
Wei Cheng ${ }^{1}$, Lin $\mathrm{Gao}^{2}$, Jie Zhang ${ }^{3}$, Jiantao $\mathrm{Lu}^{4}$ \\ ${ }^{1,3,4}$ State Key Laboratory for Manufacturing Systems Engineering, Xi' an Jiaotong University, \\ Xi' an 710049, Shaanxi, China \\ ${ }^{2}$ Institute of Biomedical Engineering, Key Laboratory of Biomedical Information Engineering of Education \\ Ministry, Xi'an Jiaotong University, Xi'an 710049, Shaanxi, China \\ ${ }^{2}$ Corresponding author \\ E-mail: 11chengw@xjtu.edu.cn, ${ }^{2}$ gaolin2013@xjtu.edu.cn,.3epicureans@163.com, \\ ${ }^{4}$ lujiantao1990@stu.xjtu.edu.cn
}

Received 20 February 2016; received in revised form 15 July 2016; accepted 19 July 2016 DOI http://dx.doi.org/10.21595/jve.2016.17432

\begin{abstract}
Acoustical signals of mechanical systems can provide original information of operating conditions, and thus benefit for machinery condition monitoring and fault diagnosis. However, acoustical signals measured by sensors are mixed signals of all the sources, and normally it is impossible to be directly used for acoustical source identification or feature extraction. Therefore, this paper presents nonlinear factor analysis (NLFA) and applies it to acoustical source separation and identification of mechanical systems. The effects by numbers of hidden neurons and mixed signals on separation performances of NLFA are comparatively studied. Furthermore, acoustical signals from a test bed with shell structures are separated and identified by NLFA and correlation analysis, and the effectiveness of NLFA on acoustical signals is validated by both numerical case studies and an experimental case study. This work can benefit for machinery noise monitoring, reduction and control, and also provide pure source information for machinery condition monitoring or fault diagnosis.
\end{abstract}

Keywords: nonlinear factor analysis, source separation and identification, feature extraction, correlation analysis, noise monitoring and control.

\section{Introduction}

Vibration and noises normally reduce the operational precision and even shorten the service life of machinery. However, vibration and noise caused by collision and friction of mechanical components can provide important information of operating conditions, and thus noise monitoring, reduction and control can be carried out based on a non-destructive measurement. In essential, measured acoustical signals are mixed signals of all sources and noises. Therefore, it has great significance to separate and identify source information from measured signals, and provides pure source information of each mechanical component especially some key components for an effective noise monitoring, reduction or control.

Generally, the measured acoustical signals are complicated and rough information of mechanical systems, which are caused by a complicated mixing of sources and transmission effects of mechanical structures. In the past decade, many researchers have devoted their efforts on transmission effects of vibration and acoustical signals. Radzevich S. P. [1] proposed an advanced technology of finishing of topologically modified pinion tooth surface for low noise/noiseless vehicle transmission. Denli H. [2] presented an optimization study of cylindrical sandwich shells to minimize the transmitted sound into the interior induced by the exterior acoustic excitations. Xin F. X. [3] formulated an analytical approach to account for the effects of mean flow on sound transmission across a simply supported rectangular aeroelastic panel. Nennig B. [4] studied the propagation of sound in a lined duct containing sheared mean flow. Bravo T. [5] described theoretical and experimental investigations into the sound absorption and transmission properties of micro-perforated panels backed by an air cavity and a thin plate. Yin J. F. [6] considered an approach using advanced statistical energy analysis that can incorporate tunneling mechanisms within a statistical approach. Fleury R. [7] studied the anomalous sound transmission 
and uniform energy squeezing through ultranarrow acoustic channels filled with zero-density metamaterials. Kim C. J. [8] developed a model for predicting the vibration transmission from two major excitation sources, ground vibration and fluid bearing force, to the tool and the workpiece position through the mechanical and control system of a precision machine. $\mathrm{Yu} \mathrm{X}$. [9] proposed a virtual panel considering an aperture as an equivalent structural component, which can be integrated with the solid/flexible structure to form a unified compound interface. Liu Y. [10] extended the prediction of sound transmission loss for a double panel structure lined with poroelastic materials to address the problem of a triple-panel structure. All these articles studied sound transmission properties through different structures, and can benefit for a passive vibration and noise control. However, to build a precise acoustical transmission model for complicated mechanical systems is still a challenging task and also costs plenty of time and resources.

Signal processing provides another way to interpret operating conditions of a system through response signals, and has benefited for system analysis, machinery condition monitoring and fault diagnosis. To effectively separate and identify acoustical sources, source separation is developed to extract source information from measured mixed signals without knowledge of sources and their mixing mode. Cheng W. studied source number estimation [11, 12], source separation [13] and source contribution evaluation $[14,15]$ methods for mechanical systems based on an effective source separation. Zhang E. L. [16] proposed an efficient solution to the separation of uncorrelated wide-band sound sources. Hioka Y. [17] proposed a method that can separate underdetermined sound sources based on a novel power spectral density estimation. Dong B. [18] offered a method for separating incoherent and compact sound sources based on the least spatial entropy. Han T. J. [19] proposed a new method of estimating the location of a predominant source in an amplitude panned stereo signal with two sources. To overcome the nonlinear mixing mode of sources in real physical systems, nonlinear factor analysis (NLFA) is developed to adaptively extract basic factors hidden in the observed signals. Mcdonald R. P. [20] firstly form a parametric function for nonlinear factor analysis in 1962, and then constructed a polynomial model with numerical methods [21]. After that, many nonlinear factor analysis methods are developed. Jochum C. [22] described a combined linear and nonlinear factor analysis program package for chemical data evaluation. Etezadiamoli J. [23] studied a 2nd generation nonlinear factor analysis. Zhu H. T. [24] presented a Bayesian analysis of a general nonlinear factor analysis model. Valpola H. [25] developed a computationally efficient algorithm for a nonlinear extension of the linear factor analysis model. Yalcin I. [26] reviewed identification ambiguity and heavy reliance on normality of nonlinear factor analysis. Besides theoretical studies, nonlinear factor analysis was also a powerful tool in real physical systems, and it has been applied to trading equity [27], structural health monitoring [28], Aliasing detection of images [29], and integrative data analysis [30]. In this paper, NLFA is applied to acoustical source separation and identification, and the separation performances of NLFA for acoustical signals are quantitatively evaluated through numerical case studies and an experimental study on a test bed with shell structures.

The remainder of this paper is organized as follows. In Section 2, basic theory and key principals of NLFA are introduced. In Section 3, separation performances of NLFA are comparatively studied with different numbers of hidden neurons and mixed signals. In Section 4, NLFA is applied to separate and identify acoustical sources based on source separation and correlation analysis. In Section 5, the conclusions of this study are summarized.

\section{Theory of nolinear factor analysis}

\subsection{Nonlinear Factor Analysis Model}

Considering a physical system of $m$ observations $\mathbf{X}(t)=\left[\mathbf{x}_{1}(t), \mathbf{x}_{2}(t), \cdots, \mathbf{x}_{m}(t)\right]^{T}, n$ sources $\mathbf{S}(t)=\left[\mathbf{s}_{1}(t), \mathbf{s}_{2}(t), \cdots, \mathbf{s}_{n}(t)\right]^{T}$, and noises $\mathbf{N}(t)=\left[\mathbf{n}_{1}(t), \mathbf{n}_{2}(t), \cdots, \mathbf{n}_{m}(t)\right]^{T}$. The mixing mode of all the sources can be expressed as a function $f()$ as a parametrised mapping from source space to observation space, which is shown in Fig. 1. Thus nonlinear factor analysis can be 
modeled about how the observations are generated from the sources [31]:

$\mathbf{x}(t)=f(\mathbf{s}(t))+\mathbf{n}(t)$

If noises can be ignored or treated as sources, the above model can be further simplified as:

$\mathbf{x}(t)=f(\mathbf{s}(t))$

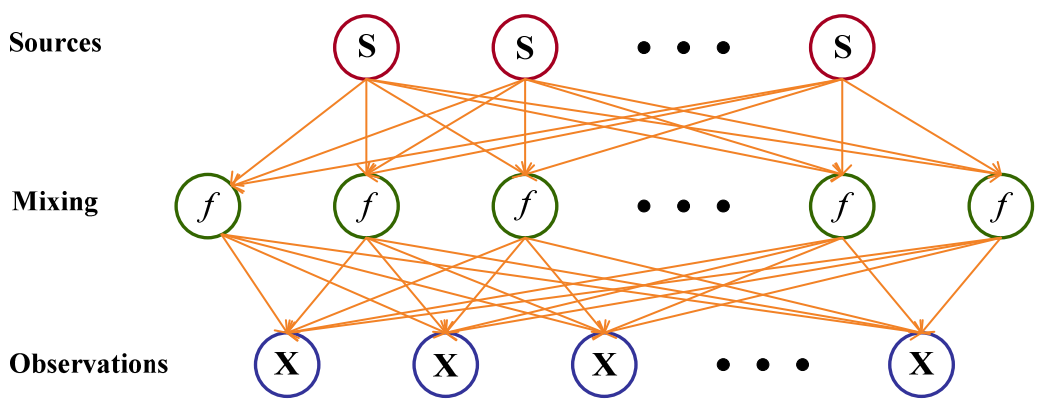

Fig. 1. The mapping from sources to observations by MLP network

In Fig. 1, the nonlinearity of each hidden neuron is the hyperbolic tangent, which is the same as the usual logistic sigmoid except for a scaling. The mapping can be defined as:

$\mathbf{x}(t)=f(\mathbf{s}(t))=\mathbf{B} \tanh (\mathbf{A s}(t)+a)+b$,

where matrices $\mathbf{A}$ and $\mathbf{B}$ are weights of first and second layer, $a$ and $b$ are corresponding biases.

The sources are assumed to have zero mean Gaussian distributions and again the variances are parametrised by $\log$-std $\mathbf{v}_{s}$, and the noise is assumed to be independent and Gaussian. Therefore, all the parameters are considered to have following distributions:

$\mathbf{x}(t) \sim N\left(f(\mathbf{s}(t)), e^{2 \mathbf{v}_{\mathbf{x}}}\right)$,

$\mathbf{s}(t) \sim N\left(0, e^{2 \mathbf{v}_{\mathbf{s}}}\right)$,

$\mathbf{A} \sim N(0,1)$,

$\mathbf{B} \sim N\left(0, e^{2 \mathbf{v}_{\mathbf{B}}}\right)$,

$a \sim N\left(m_{a}, e^{2 \mathbf{v}_{a}}\right)$

$b \sim N\left(m_{b}, e^{2 \mathbf{v}_{b}}\right)$,

$\mathbf{v}_{\mathbf{x}} \sim N\left(m_{\mathbf{v}_{\mathbf{x}}}, e^{2 \mathbf{v}_{\mathbf{v}_{\mathbf{x}}}}\right)$

$\mathbf{v}_{\mathbf{s}} \sim N\left(m_{\mathbf{v}_{\mathbf{s}}}, e^{2 \mathbf{v}_{\mathbf{v}_{\mathbf{s}}}}\right)$,

$\mathbf{v}_{B} \sim N\left(m_{\mathbf{v}_{B}}, e^{2 \mathbf{v}_{\mathbf{v}_{B}}}\right)$.

The prior distributions of $m_{a}, \mathbf{v}_{a}, m_{b}, \mathbf{v}_{b}$, the six hyper-parameters $m_{\mathbf{v}_{\mathbf{s}}}, \ldots, m_{\mathbf{v}_{B}}$ are assumed to be Gaussian with zero mean and standard deviation, and the priors are assumed to be very flat.

\subsection{Objective function}

If there are only the observations $\mathbf{X}$, the posterior pdf of all the hidden sources should be approximated. Thus the objective function measures the misfit between the actual posterior pdf $p(\mathbf{S} \mid \mathbf{X})$ and its approximation $q(\mathbf{S} \mid \mathbf{X})$ :

$q(\mathbf{S} \mid \mathbf{X})=\prod_{i} q\left(\mathbf{s}_{i} \mid \mathbf{X}\right)$. 
Each individual approximation $q\left(\mathbf{s}_{i} \mid \mathbf{X}\right)$ is parametrised by the posterior mean $\overline{\mathbf{S}}_{i}$ and variance $\tilde{\mathbf{S}}_{i}$ of the parameter. The objective function $C_{\mathbf{S}}(\mathbf{X} ; \overline{\mathbf{S}} ; \tilde{\mathbf{S}})$ contains $C_{q}(\mathbf{X} ; \mathbf{S})$ and $C_{p}(\mathbf{X} ; \mathbf{S})$ :

$C_{\mathbf{S}}(\mathbf{X} ; \overline{\mathbf{S}}, \tilde{\mathbf{S}})=C_{q}(\mathbf{X} ; \mathbf{S})+C_{p}(\mathbf{X} ; \mathbf{S})$,

$C_{q}(\mathbf{X} ; \mathbf{S})=\ln q(\mathbf{S} \mid \mathbf{X})=\sum_{i}-\frac{1}{2} \ln 2 \pi e \tilde{\mathbf{s}}_{i}$,

$C_{p}(\mathbf{X} ; \mathbf{S})=-\ln p(\mathbf{X}, \mathbf{S})=\frac{1}{2}\left(\mathbf{S}-m_{\mathbf{S}}\right)^{2} e^{-2 \mathbf{V}_{\mathbf{S}}}+\mathbf{V}_{\mathbf{S}}+\frac{1}{2} \ln 2 \pi$.

Since $\mathbf{S}, m_{\mathbf{S}}, \mathbf{V}_{\mathbf{S}}$ are independent in $q$, the expectation over $q$ produces:

$\frac{1}{2}\left[\left(\overline{\mathbf{S}}-\bar{m}_{\mathbf{S}}\right)^{2}+\widetilde{\mathbf{S}}+\widetilde{m}_{\mathbf{S}}\right] e^{2 \widetilde{\mathbf{V}}_{\mathbf{S}}-2 \overline{\mathbf{V}}_{\mathbf{S}}}+\overline{\mathbf{V}}_{\mathbf{S}}+\frac{1}{2} \ln 2 \pi$.

From Eq. (4), there has:

$-\ln p(\mathbf{X})=\frac{1}{2}(\mathbf{X}-f)^{2} e^{-2 \mathbf{V}_{\mathbf{X}}}+\mathbf{V}_{\mathbf{X}}+\frac{1}{2} \ln 2 \pi$,

$E(q)=\frac{1}{2}\left[(\mathbf{X}-\bar{f})^{2}+\tilde{f}\right] e^{-2 \widetilde{\mathbf{V}}_{\mathbf{X}}-2 \overline{\mathbf{V}}_{\mathbf{X}}}+\overline{\mathbf{V}}_{\mathbf{X}}+\frac{1}{2} \ln 2 \pi$,

$\bar{f}\left(\mathbf{x}_{i}\right) \approx \bar{f}\left(\overline{\mathbf{x}}_{i}\right)+\frac{1}{2} f^{\prime \prime}\left(\overline{\mathbf{x}}_{i}\right) \tilde{\mathbf{x}}_{i}$,

$\tilde{f}\left(\mathbf{x}_{i}\right)=\tilde{f}^{*}\left(\mathbf{x}_{i}\right)+\sum_{i} \tilde{\mathbf{s}}_{i}\left[\frac{\partial f\left(\mathbf{x}_{i}\right)}{\partial \mathbf{s}_{i}}\right]^{2}$,

where $\tilde{f}\left(\mathbf{x}_{i}\right)$ denotes the total posterior variance of the output $f\left(\mathbf{x}_{i}\right)$, and $\tilde{f}^{*}\left(\mathbf{x}_{i}\right)$ denotes the variance originating from the weights and biases.

\subsection{Parameter updating rules}

After the objective function is constructed, the next step is using optimization algorithm to minimize the $C_{\mathbf{S}}(\mathbf{X} ; \overline{\mathbf{S}}, \tilde{\mathbf{S}})$ with respect to the posterior means $\overline{\mathbf{S}}$ and variances $\tilde{\mathbf{S}}$ of the unknown variables. The fixed point equation is used to update the variances:

$\frac{\partial C}{\partial \widetilde{\mathbf{s}}}=\frac{\partial C_{p}}{\partial \widetilde{\mathbf{s}}}+\frac{\partial C_{q}}{\partial \widetilde{\mathbf{s}}}=\frac{\partial C_{p}}{\partial \widetilde{\mathbf{s}}}-\frac{1}{2 \tilde{\mathbf{s}}} \Rightarrow \widetilde{\mathbf{s}}=\frac{1}{2 \frac{\partial C_{q}}{\partial \widetilde{\mathbf{s}}}}$.

Newton iteration is used as posterior variances $\tilde{\mathbf{S}}$ containing the information about the second order derivatives of the objective function $C_{\mathbf{S}}(\mathbf{X} ; \overline{\mathbf{S}}, \tilde{\mathbf{S}})$, and there has:

$\tilde{\mathbf{s}} \leftarrow \frac{1}{\left[2 \frac{\partial^{2} C_{\mathbf{s}}}{\partial \overline{\mathbf{s}}^{2}}\right]^{\alpha_{\tilde{\mathbf{s}}}}} \tilde{\mathbf{s}}^{1-\alpha_{\tilde{\mathbf{s}}}}$,

$\overline{\mathbf{s}} \leftarrow \overline{\mathbf{s}}-\alpha_{\overline{\mathbf{s}}} \frac{\partial C_{p}}{\partial \overline{\mathbf{s}}} \widetilde{\mathbf{s}}$,

where $\alpha_{\overline{\mathbf{s}}}$ is a learning parameter to dampen the oscillations in the fixed point algorithms according to the following rule: 
$\alpha_{\overline{\mathbf{s}}} \leftarrow \begin{cases}0.8 \alpha_{\overline{\mathbf{s}}}, & \text { sign of change is different, } \\ \min \left(1,1.05 \alpha_{\overline{\mathbf{s}}}\right), & \text { sign of change is same. }\end{cases}$

\subsection{Source Identification}

As some information of sources for mechanical systems normally can be obtained by the theory studies or Instructions, a waveform correlation is constructed to identify the source information by the correlation analysis between the sources and the separated signals. For discrete signals $\mathbf{s}(t)$ and $\mathbf{y}(t)$, the waveform correlation coefficient $\rho_{W}$ is defined as:

$\rho_{W}=\frac{\max R_{\text {sy }}(k)}{\sqrt{\max _{\mathbf{s s}}(k) \times \max R_{\mathbf{y y}}(k)}}, \quad 0 \leq k \leq T$,

where:

$R_{\mathbf{s y}}(k)=\sum_{i=0}^{T-k-1} \mathbf{s}(i+k) \mathbf{y}(i), \quad R_{\mathbf{s s}}(k)=\sum_{i=0}^{T-k-1} \mathbf{s}(i+k) \mathbf{s}(i)$,
$R_{\mathbf{y y}}(k)=\sum_{i=0}^{T-k-1} \mathbf{y}(i+k) \mathbf{y}(i), \quad \forall k \geq 0$,

and $T$ is the data length.

\section{Numerical case study}

\subsection{Introductions}

In this section, typical signals of mechanical systems are artificially generated, which are used to test the effects by numbers of hidden neurons and mixed signals on the separation performances of NLFA. Since acoustical signals transmit from sources to measured points mainly through air, and the generating mechanisms of sources are statistically different. Therefore, all the sources are considered to be linearly mixed and independent from each other.

Source signals are: signal $\mathbf{s}_{1}(t)$ is a periodic wave of oscillating attenuation that simulates mechanical shocks; signal $\mathbf{s}_{2}(t)$ is a sinusoidal wave that simulates a vibration signal of rotational equipment; signal $\mathbf{s}_{3}(t)$ is a periodic wave that simulates amplitude modulation; signal $\mathbf{s}_{4}(t)$ is a white noise that simulates environmental noises. In the numerical case study, the noise is considered to be one source, and the generating functions of the sources are listed as follows:

$\left.\mathbf{S}(t)=\left[\begin{array}{l}\mathbf{s}_{1}(t) \\ \mathbf{s}_{2}(t) \\ \mathbf{s}_{3}(t) \\ \mathbf{s}_{4}(t)\end{array}\right]=\left[\begin{array}{c}\sin (200(t-0.125 n-0.025)) \\ \exp (-20(t-0.125 n-0.025)) u(t-0.125 n-0.025)\end{array}\right)\right]$,

where $u(t)$ is a step function.

Generally, the measured signals are mixed signals of all the sources. Therefore, linear superposition is applied to artificially produce the mixed signals, and the mixing matrix $\mathbf{A}$ is randomly generated as shown in the following matrix: 
$\mathbf{A}=\left[\begin{array}{cccc}-0.55 & 0.85 & -0.73 & 0.61 \\ 0.71 & -0.51 & 0.44 & -0.57 \\ 0.68 & -0.71 & -0.68 & 0.72 \\ -0.54 & 0.65 & 0.62 & -0.43\end{array}\right]$

The waveforms of source signals and mixed signals are shown in Fig. 2 and Fig. 3, respectively. Obviously, from Fig. 2 it can be seen that all the source signals have typical wave features. However, from Fig. 3 it is very difficult to identify the features of the sources as all the waveforms of sources are coupling together. Therefore, nonlinear factor analysis is applied to separate the mixed signals into uncorrelated factors, and then source identification can be carried out by a correlation analysis between the separated factors and the given sources.

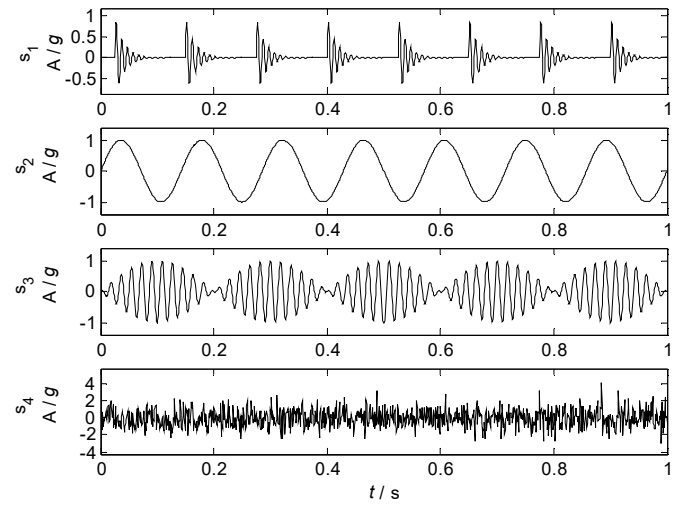

Fig. 2. Waveforms of source signals

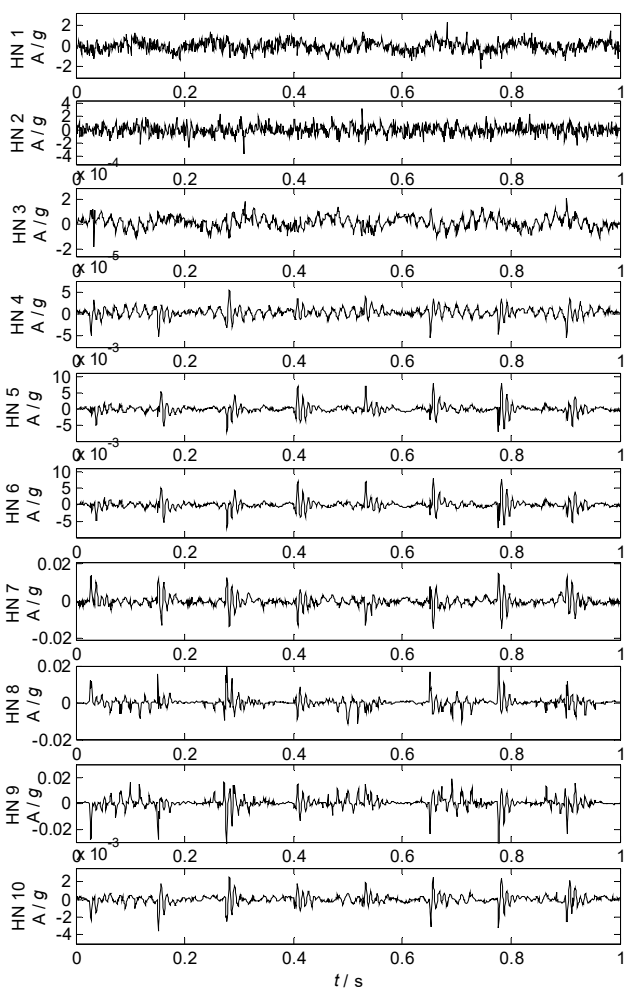

Fig. 4. Separated factor 1 by NLFA $\times \frac{0}{2} \int_{-5}^{5}\left[\begin{array}{ccc}1 \\ 0\end{array}\right.$

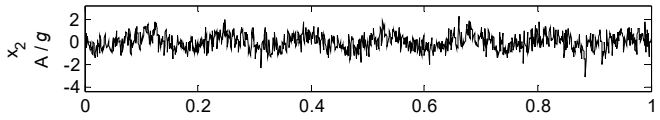

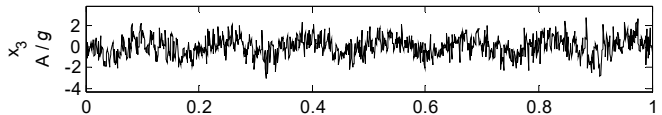

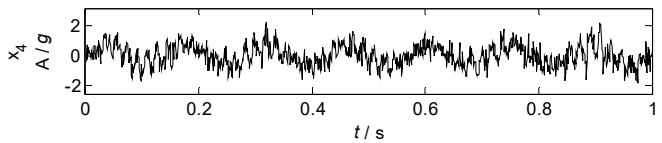

Fig. 3. Waveforms of mixed signals
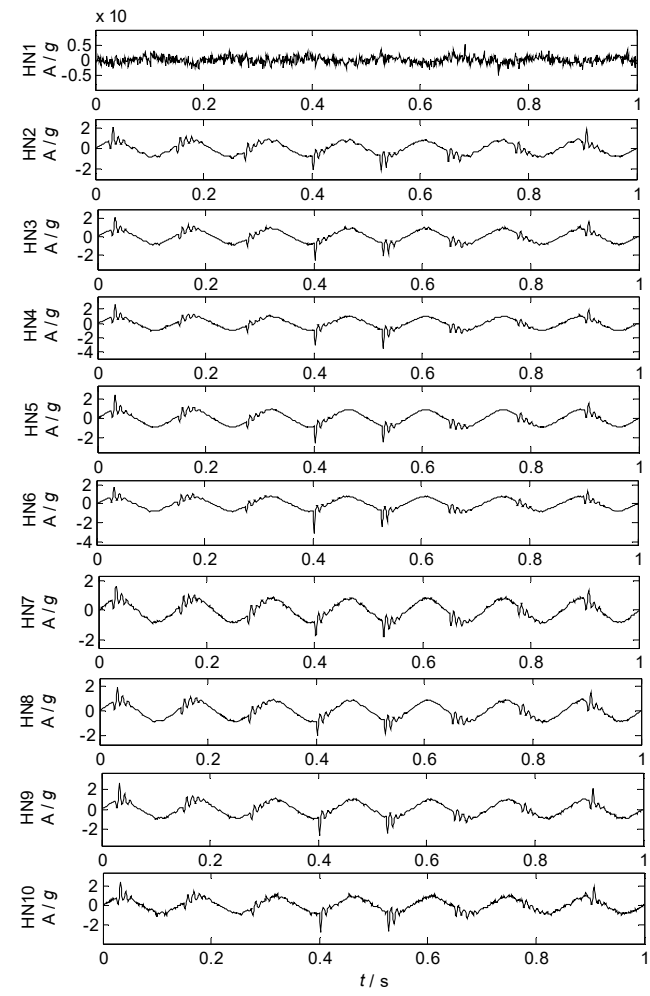

Fig. 5. Separated factor 2 by NLFA 


\subsection{Effects by numbers of hidden neurons}

To test the effects by numbers of hidden neurons $(\mathrm{HN})$, four mixed signals are separated into four separated factors using nonlinear factor analysis with different numbers of hidden neurons, and the waveforms of each separated component in different separating conditions are shown in Fig. 4-7.

Fig. 4-7 show the waveforms of different separated factors by NLFA with different HN: Fig. 4 shows that the major feature of source 1 can be extracted as $\mathrm{HN}$ is no less than 4, Fig. 5 shows that the major features of source 2 are extracted as $\mathrm{HN}$ is no less than 2, Fig. 6 shows that the major features of source 3 can be extracted as $\mathrm{HN}$ is no less than 3, and Fig. 7 shows that the major feature of source 4 can be extracted as $\mathrm{HN}$ is no less than 2. Generally, the separation performances of NLFA become better as the number of $\mathrm{HN}$ increases $(\mathrm{HN}<4)$, and then the separation performances change very little as $\mathrm{HN}$ increases $(\mathrm{HN} \geq 4)$.

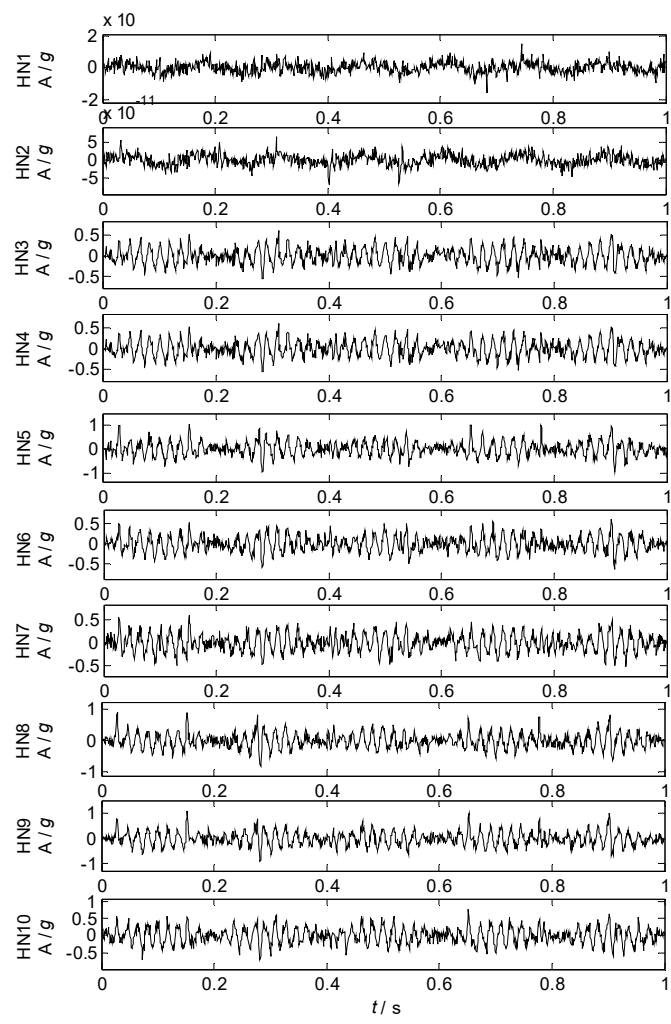

Fig. 6. Separated factor 3 by NLFA

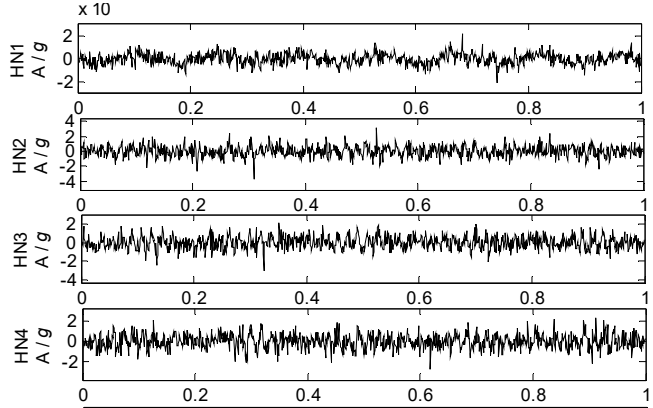

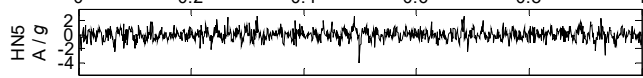

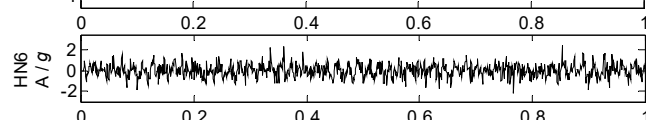

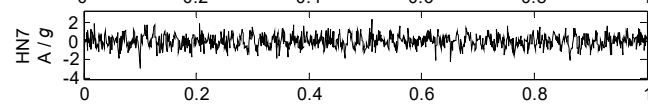

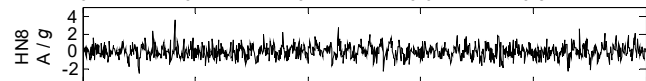

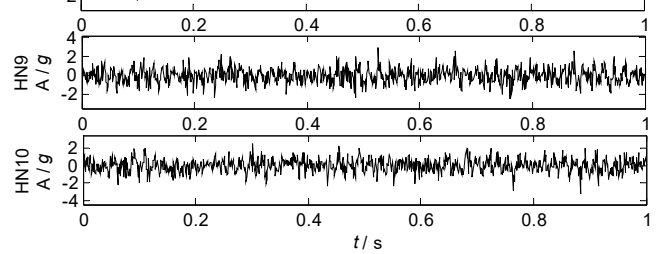

Fig. 7. Separated factor 4 by NLFA

The waveform correlation coefficients between the separated components and related sources are shown in Fig. 8: the separation performances become better as the number of $\mathrm{HN}$ increases $(\mathrm{HN} \leq 5)$, and change very litter $(\mathrm{HN}>5)$, the source 2 and source 4 can be well separated as $\mathrm{HN}$ is only 2 . Generally, increasing the number of $\mathrm{HN}$ can improve the separation performances, but it also costs more calculating time (Cost time: $15.44 \mathrm{~s}(\mathrm{HN} 2), 16.09 \mathrm{~s}$ (HN3), $16.35 \mathrm{~s}$ (HN4), 17.25 s (HN5), $17.70 \mathrm{~s}$ (HN6), $18.22 \mathrm{~s}$ (HN7), $19.60 \mathrm{~s}$ (HN8), $21.90 \mathrm{~s}$ (HN9), $22.26 \mathrm{~s}$ (HN10)). Moreover, as the number of $\mathrm{HN}$ increases, there also can cost calculating errors which can be found at the curve of separated factor 1 in Fig. 8. Therefore, normally the number of HN should be as less as possible to improve the calculating efficiency and avoid additional calculating errors. From Fig. 8, the number of $\mathrm{HN}$ is suggested to be 5-7. 


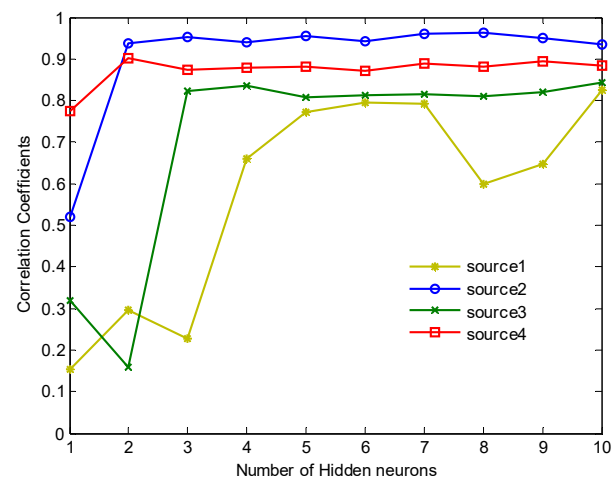

Fig. 8. Waveform correlation coefficients

\subsection{Effects by numbers of mixed signals}

To test the effects by numbers of mixed signals, the number $m$ of mixed signals are set from 2 to 10 , and each mixed signals are generated by all the sources with a randomly mixing matrix. Then the mixed signals are separated by NLFA, and the waveforms of separated components in different conditions are shown in Fig. 9-12.
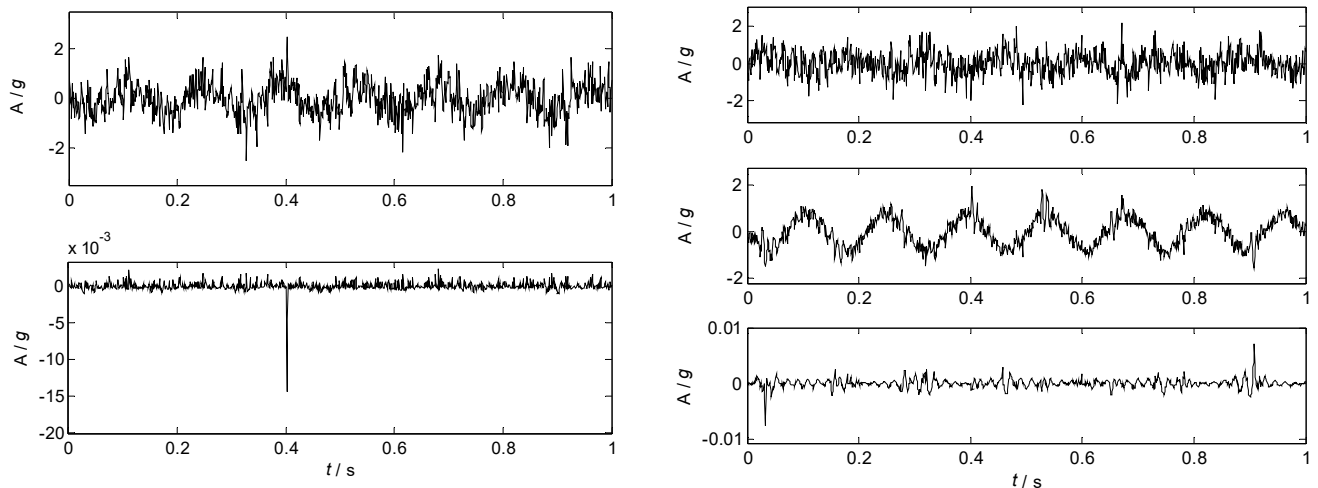

Fig. 9. Separated components $(m=2)$
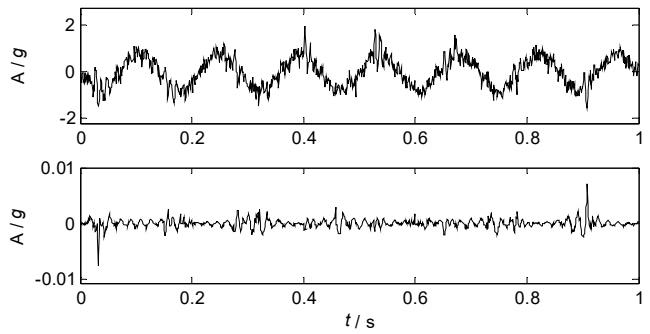

Fig. 10. Separated components $4(m=3)$
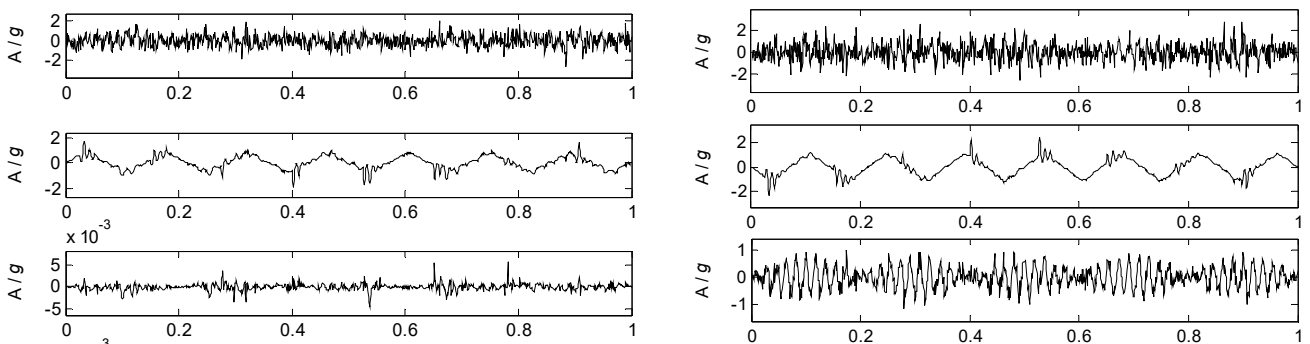

$\times 10^{-3}$

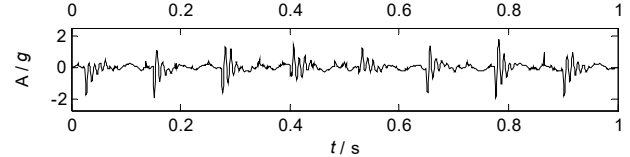

Fig. 12. Separated components $(m=5)$

Fig. 11. Separated components $(m=4)$

Fig. 9-12 show the waveforms of separated factors as the numbers of the mixed signals are 2, 3,4 and 5 respectively. Fig. $9(m=2)$ shows that 2 factors are separated from two mixed signals, and only a noisy sine wave but no other source feature is separated. Fig. $10(\mathrm{~m}=3)$ shows that 3 
components are separated from 3 mixed signals, and only the sine wave are well separated. Fig. 11 shows that 4 components are separated from 4 mixed signals, and the major features of source 2 and source 4 are well separated. The features of source 1 and source 3 are separated, but the features are not clear as they are still coupling with other waveforms. Fig. 12 shows that 4 components are separated from 5 mixed signals, and the major features of all the sources are well separated. Therefore, in this case study, the separation performances of NLFA become better as the number of the mixed signals increases.

To quantitatively reveals effects by the number of the mixed signals, more numerical case studies are provided, and correlation coefficients between the separated factors and the sources are shown in Fig. 13 as the numbers of the mixed signals are from 2 to 10. From Fig. 13, it can be seen that the separation performances increase fast $(m<4)$, and changes very little $(m \geq 4)$. Therefore, increasing the number of the mixed signals can improve the separation performances from theory analysis. However, in engineering applications, more mixed signals mean that more noises may be added, and the effectiveness of separation cannot be guaranteed. Therefore, it is suggested the number of mixed signals should be equal to the number of sources, or more than one. In this case study, the number of the mixed signals is suggested to be 4 or 5 .

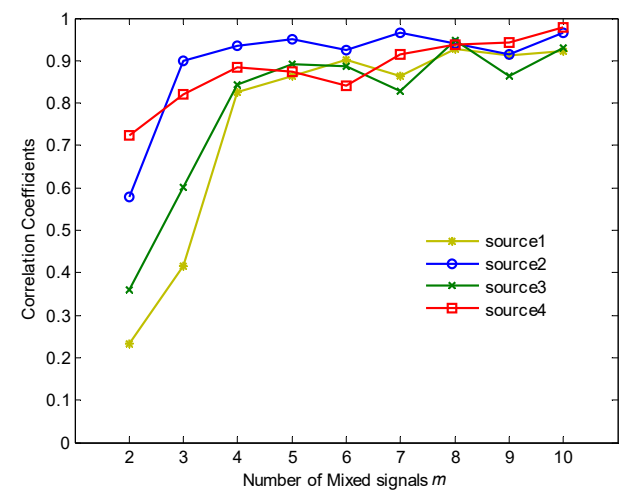

Fig. 13. Waveform correlation coefficients

\section{Experimental study}

\subsection{Introductions of the test bed}

A test bed is constructed to test the separation performances of NLFA, which has four major components: two end covers, a shell structure, two clapboards, and supports. Rubber air springs support the whole test bed, which can reduce the effects of ground vibrations and environmental noises. Three acoustical sources are designed in the test bed: two loudspeakers controlled by signal generators, and one motor controlled by a frequency converter. The structure of the test bed is shown in Fig. 14.

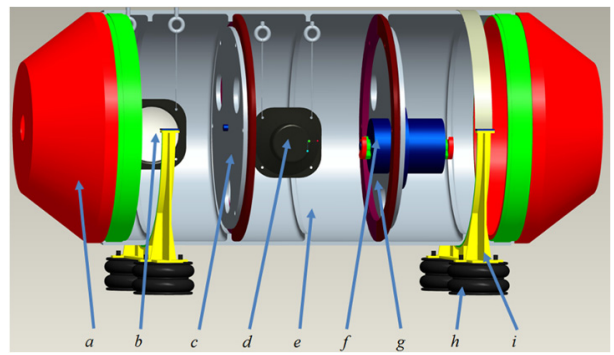

Fig. 14. The structure of the test bed: a) End cover; b) Loudspeaker I; c) Left clapboard; d) Loudspeaker II; e) Shell; f) Motor; g) Right clapboard; h) Rubber springs; i) Supports 


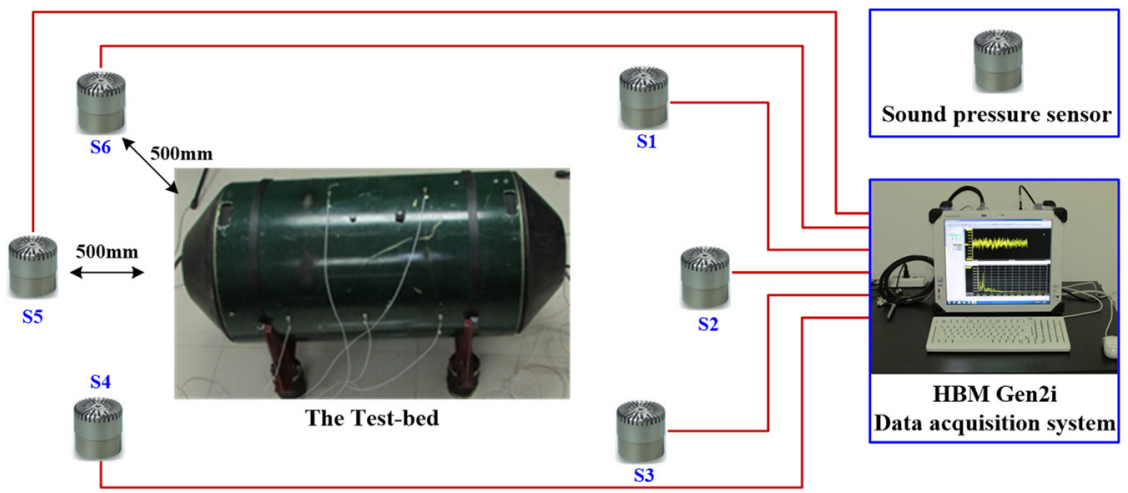

Fig. 15. The framework of data acquisition system

Six sound pressure sensors are used to collect acoustical signals, and they are placed in six different directions of the test bed with a same distance of 500 millimeters. HBM Gen2i data acquisition system is used to collect acoustical data. The framework of the data acquisition system is shown in Fig. 15, and the test parameters are shown in Table 1.

Table 1. The test parameters of the data acquisition system

\begin{tabular}{|c|c|}
\hline Parameters & Values and Units \\
\hline Sound pressure sensors & 6 \\
\hline HBM Gen2i Data acquisition system & 1 \\
\hline Test bed with shell structures & 1 \\
\hline Sampling frequency & $10240 \mathrm{~Hz}$ \\
\hline Sampling length & $10 \mathrm{~seconds}$ \\
\hline Frequency of Loudspeaker I & $1500 \mathrm{~Hz}$ \\
\hline Frequencies of Loudspeaker II & $180020002200 \mathrm{~Hz}$ \\
\hline Rotational speed of motor & $1200 \mathrm{r} / \mathrm{min}(20 \mathrm{~Hz})$ \\
\hline
\end{tabular}

\subsection{Acoustical signals of the test bed}

As all the sources are working with the given parameters, the mixed acoustical signals are measured by six sound pressure sensors. In the experimental study, the acoustical signals from sensors 2, 3, 4 and 6 are used as mixed signals. Generally, the number of the mixed signals is 1 more than that of sources, which is to guarantee an effective source separation of NLFA and also reduce noise effects of environment, and the directions of these sensors represent a diversified mixing mode of sources.
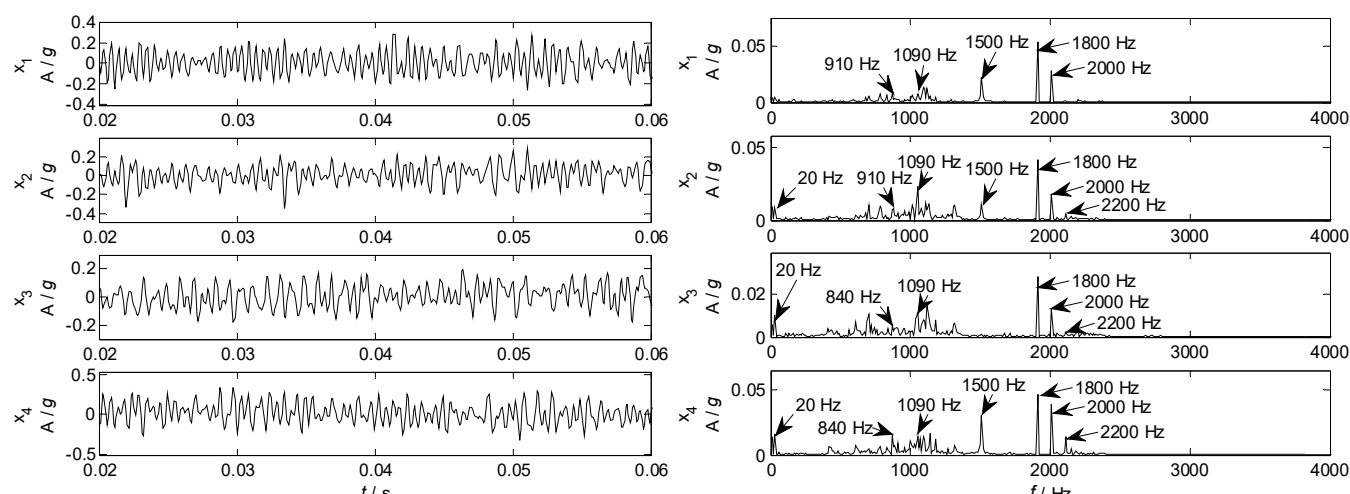

Fig. 16. Waveforms of mixed signals

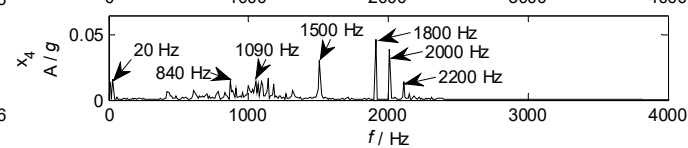

Fig. 17. Spectrums of mixed signals 
The waveforms and spectrums of mixed signals are shown in Fig. 16 and Fig. 17 respectively. From waveforms in Fig. 16, it is very difficult to identify source features except some periodic waves. While from spectrums in Fig. 17, nearly all the mixed signals contain some major components of $20,910,1090,1500,1800,2000$, and $2200 \mathrm{~Hz}$, which can be used to identify sources. Generally, independent source information cannot be directly identified from mixed signals as they are coupling together.

\subsection{Acoustical source separation}

NLFA is applied to separate mixed signals into uncorrelated factors, and 3 factors are extracted from the given mixed signals, whose waveforms and spectrums are shown in Fig. 18 and Fig. 19, respectively. Fig. 18 clearly shows that the waveform of the separated factor $\mathbf{y}_{1}$ has clearly periodic features, and the basic component is a sine wave. The spectrum in Fig. 19 also shows that the separated factor $\mathbf{y}_{1}$ has a major component of $1500 \mathrm{~Hz}$. The waveform of the separated component $\mathbf{y}_{2}$ has a typical feature of a sine wave with amplitude modulation, whose spectrum also clearly shows typical features of 1800,2000 , and $2200 \mathrm{~Hz}$. The waveform of the separated factor $\mathbf{y}_{3}$ has an unclear feature of a sine wave with randomly amplitude modulation, whose spectrum has 4 major components of 20, 870, 910, and $1090 \mathrm{~Hz}$.
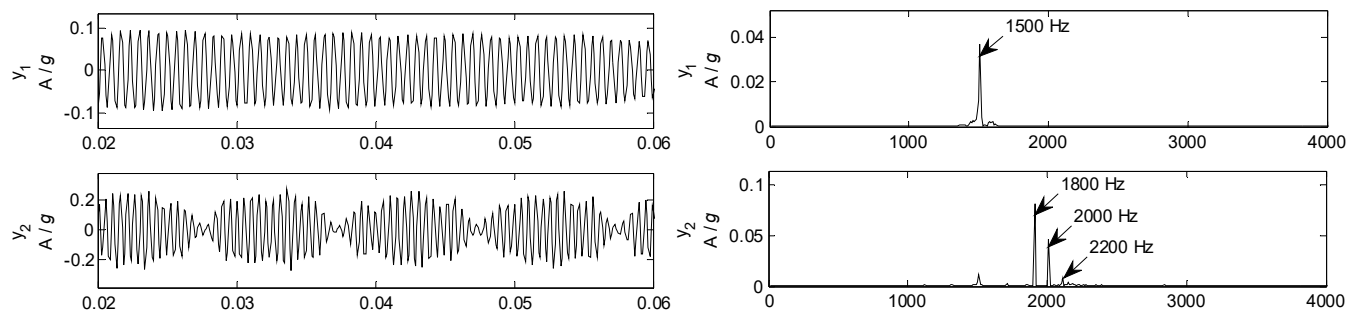

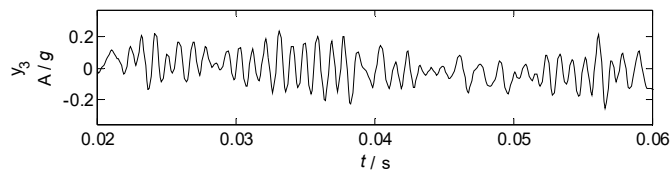

Fig. 18. Waveforms of separated factors

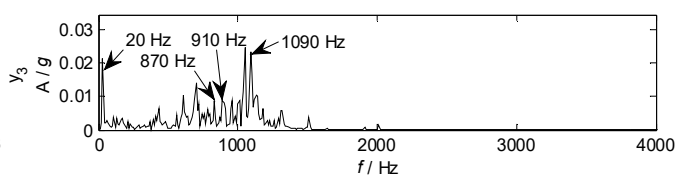

Fig. 19. Spectrums of separated factors

Comparing the spectrums of separated components with parameters of experimental setups, it can be speculated that the separated factor $\mathbf{y}_{1}$ represents a typical feature of source 1 from Loudspeaker I, while the separated factors $\mathbf{y}_{2}$ and $\mathbf{y}_{3}$ represent typical features of source 2 and 3 from loudspeaker II and the motor, respectively. However, this is just based on parameters of experimental setups, and still not a convincing and reliable source identification method.

\subsection{Acoustical source identification and validation}

To intelligently identify sources from the separated factors, and validate the effectiveness of NLFA for acoustical signals of real mechanical systems, source signals are measured independently by the closest sensors in the condition that only the related source is working with given parameters. The waveforms of independent sources from sensors 1, 4, and 6 for Loudspeaker I, Loudspeaker II, and the motor are shown in Fig. 20, and their corresponding spectrums are shown in Fig. 21.

Comparing waveforms in Fig. 20 and spectrums in Fig. 21 of source signals with that in Fig. 18 and Fig. 19 of separated factors, the waveforms of separated factors are very similar to that of related sources: the separated factor $\mathbf{y}_{1}$ and source $\mathbf{s}_{1}$ have a clear sine wave of $1500 \mathrm{~Hz}$, which well agrees with the experimental setup $(1500 \mathrm{~Hz})$ of Loudspeaker I; the separated factor $\mathbf{y}_{2}$ and source $\mathbf{s}_{2}$ have 3 major components of 1800,2000 , and $2200 \mathrm{~Hz}$ corresponding to the 
experimental setup of Loudspeaker II; the separated factor $\mathbf{y}_{3}$ and source $\mathbf{s}_{3}$ have a basic sine wave $(20 \mathrm{~Hz})$ with an uncertainty amplitude modulation, which can be interpreted as an eccentric vibration of the motor, and the uncertainty amplitude modulation $(840,910,1090 \mathrm{~Hz}) \mathrm{can}$ be caused by rubbing. Therefore, all the major components of source information have been effectively separated from mixed signals by NLFA.
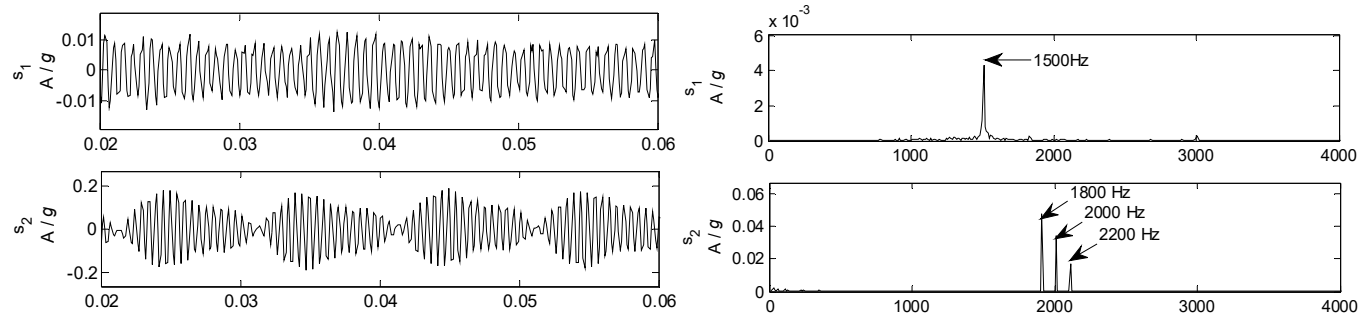

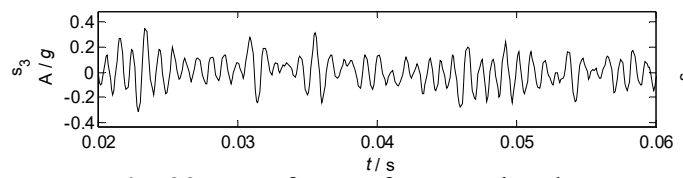

Fig. 20. Waveforms of source signals

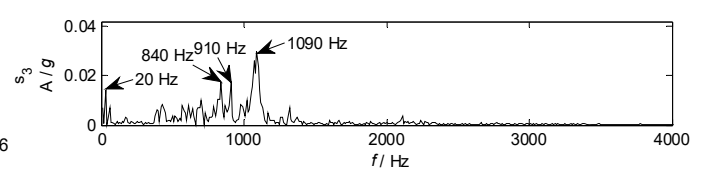

Fig. 21. Spectrums of source signals

To quantitatively and intelligently identify sources from separated factors, waveform correlation analysis is used to measure similarity between separated factors and sources. All 3 separated factors are made correlation analysis with all 3 sources, and their correlation coefficients are listed in the correlation matrix $\Omega_{W}$ :

$\Omega_{W}=\left[\begin{array}{lll}0.92 & 0.01 & 0.03 \\ 0.13 & 0.92 & 0.07 \\ 0.02 & 0.01 & 0.72\end{array}\right]$

The correlation matrix $\Omega_{W}$ shows that correlation coefficients between separated factors and related sources are $0.92,0.92$ and 0.72 , which indicate high correlation coefficients or high similarity between separated factors and related sources (Liu [32] obtained waveform correlation coefficients of $0.77 \pm 0.03$ for ECG signals with noises, and Farila [33] obtained waveform correlation coefficients of $0.70 \pm 0.09$ for non-stationary surface myoelectric signals); while correlation coefficients between separated factors and unrelated sources are all less than 0.13 , which indicates that all 3 sources have a good independence property. Therefore, a threshold $\gamma$ can be set as $\gamma \in(0.13,0.72)$ (Normally $0.55 \sim 0.65)$ to intelligently identify acoustical sources. Furthermore, high correlation coefficients between separated factors and related sources validate that the effectiveness of NLFA in acoustical source separation and identification.

\section{Conclusions}

This paper presents fundamental theory and key principals of nonlinear factor analysis, and validates the effectiveness of NLFA according to numerical case studies and an experimental study on a test bed with shell structures. All the case studies indicate that the acoustical sources can be effectively separated and intelligently identified.

In numerical case studies, separation performances of NLFA are tested in different conditions. For cases with different numbers of hidden neurons, separation performances of NLFA improve greatly as the number of hidden neurons is under 4 , and change very little above 4 , which mean that the optimal number of hidden neurons is 4 . For cases with different numbers of mixed signals, the major waveform information of sources is well separated until the number of mixed signals is above 4, and separation performances of NLFA changes very little as the number of mixed signals 
increases, which mean that the optimal number of mixed signals is 4 or 5 . Generally, increasing the numbers of hidden neurons and mixed signals can improve separation performances of NLFA. However, more observations and hidden neurons can not only cause more noises and calculating errors, but also cost a lot of calculating time. Therefore, the numbers of hidden neurons and mixed signals should be kept as small as possible. In the experimental study on a test bed, all the correlation coefficients between the separated factors and sources are more than 0.72 , which also indicates an effective acoustical source separation. If artificially set a threshold $\gamma \in(0.55,0.65)$ for correlation coefficients, all the acoustical sources can be intelligently identified.

This work can benefit for noise monitoring, reduction and control, and provide pure source information for machinery condition monitoring and fault diagnosis.

\section{Acknowledgements}

This work was supported by the projects of National Nature Science Foundation of China (No. 51305329), the China Postdoctoral Science Foundation (No. 2014T70911), the Doctoral Foundation of Education Ministry of China (No. 20130201120040), and Basic Research Project of Natural Science in Shaanxi Province (No. 2015JQ5183).

\section{References}

[1] Radzevich S. P. Technological methods for noise/vibration reduction in driveline/transmission of trucks and all-wheel-drive vehicles. International Journal of Vehicle Noise and Vibration, Vol. 2, Issue 4, 2006, p. 283-291.

[2] Denli H., Sun J. Q. Structural-acoustic optimization of sandwich cylindrical shells for minimum interior sound transmission. Journal of Sound and Vibration, Vol. 316, Issue 1, 2008, p. 32-49.

[3] Xin F. X., Lu T. J. Analytical modeling of sound transmission across finite aeroelastic panels in convected fluids. Journal of the Acoustical Society of America, Vol. 128, Issue 3, 2010, p. 1097-1107.

[4] Nennig B., Ben Tahar M., Perrey-Debain E. A displacement-pressure finite element formulation for analyzing the sound transmission in ducted shear flows with finite poroelastic lining. Journal of the Acoustical Society of America, Vol. 130, Issue 1, 2011, p. 42-51.

[5] Bravo T., Maury C., Pinhede C. Sound absorption and transmission through flexible micro-perforated panels backed by an air layer and a thin plate. Journal of the Acoustical Society of America, Vol. 131, Issue 5, 2012, p. 3853-3863.

[6] Yin J. F., Hopkins C. Prediction of high-frequency vibration transmission across coupled, periodic ribbed plates by incorporating tunneling mechanisms. Journal of the Acoustical Society of America, Vol. 133, Issue 4, 2013, p. 2069-2081.

[7] Fleury R., Alu A. Extraordinary sound transmission through density-near-zero ultranarrow channels. Physical Review Letters, Vol. 111, Issue 5, 2013, p. 055501.

[8] Kim C. J., Oh J. S., Park C. H. Modelling vibration transmission in the mechanical and control system of a precision machine. CIRP Annals Manufacturing Technology, Vol. 63, Issue 1, 2014, p. 349-352.

[9] Yu X., Cheng L., Guyader J. L. On the modeling of sound transmission through a mixed separation of flexible structure with an aperture. Journal of the Acoustical Society of America, Vol. 135, Issue 5, 2014, p. 2785-2796.

[10] Liu Y. Sound transmission through triple-panel structures lined with poroelastic materials. Journal of Sound and Vibration, Vol. 339, 2015, p. 376-395.

[11] Cheng W., Lee S., Zhang Z. S., He Z. J. Independent component analysis based source number estimation and its comparison for mechanical systems. Journal of Sound and Vibration. Vol. 331, Issue 23, 2012, p. 5153-5167.

[12] Cheng W., Zhang Z. S., Cao H. R., et al. A comparative study of information-based source number estimation methods and experimental validations on mechanical systems. Sensors, Vol. 14, Issue 5, 2014, p. 7625-7646.

[13] Cheng W., Zhang Z. S., Lee S., He Z. J. Investigations of denoising source separation technique and its application to source separation and identification of mechanical vibration signals. Journal of Vibration and Control, Vol. 20, Issue 14, 2014, p. 2100-2117. 
[14] Cheng W., He Z. J., Zhang Z. S. A comprehensive study of vibration signals for a thin shell structure using enhanced independent component analysis and experimental validation. Journal of Vibration and Acoustics - Transactions of the ASME. Vol. 136, Issue 4, 2014.

[15] Cheng W., Zhang Z. S., Lee S., He Z. J. Source contribution evaluation of mechanical vibration signals via enhanced independent component analysis. Journal of Manufacturing Science and Engineering-Transactions of the ASME, vol. 134, Issue 2, 2012.

[16] Zhang E. L., Antoni J., Dong B., Snoussi H. Bayesian space-frequency separation of wide-band sound sources by a hierarchical approach. Journal of the Acoustical Society of America, Vol. 132, Issue 5, 2012, p. 3240-3250.

[17] Hioka Y., Furuya K., Kobayashi K., Niwa K., Haneda Y. Underdetermined sound source separation using power spectrum density estimated by combination of directivity gain. IEEE Transactions on Audio Speech and Language Processing, Vol. 21, Issue 5, 2013, p. 1240-1250.

[18] Bin D., Antoni J., Erliang Z. Blind separation of sound sources from the principle of least spatial entropy. Journal of Sound and Vibration, Vol. 333, Issue 9, 2014, p. 2643-2668.

[19] Han T. J., Kim K. J., Park H. Location estimation of predominant sound source with embedded source separation in amplitude-panned stereo signal. IEEE Signal Processing Letters, Vol. 22, Issue 10, 2015, p. 1685-1688.

[20] McDonald R. P. A general-approach to nonlinear factor-analysis. Psychometrika, Vol. 27, Issue 4, 1962, p. 397-415.

[21] McDonald R. P. Numerical methods for polynomial models in nonlinear factor analysis. Psychometrika, Vol. 32, Issue 2, 1967, p. 77-77.

[22] Jochum C., Kowalski B. R. A combined linear and nonlinear factor analysis program package for chemical data evaluation. Analytica Chimica ACTA, Computer Techniques and Optimization, Vol. 133, Issue 4, 1981, p. 583-590.

[23] Etezadiamoli J., McDonald R. P. A 2nd generation nonlinear factor-analysis. Psychometrika, Vol. 48, 1983, p. 315-342.

[24] Zhu H. T., Lee S. Y. Statistical analysis of nonlinear factor analysis models. British Journal of Mathematical and Statistical Psychology, Vol. 52, Issue 2, 1999, p. 225-242.

[25] Valpola H. Bayesian ensemble learning for nonlinear factor analysis. ACTA Polytechnica Scandinavica, Mathematics and Computing Series, 2000, p. 6-54.

[26] Yalcin I., Amemiya Y. Nonlinear factor analysis as a statistical method. Statistical Science, Vol. 16, Issue 2, 2001, p. 275-294.

[27] Jones C. S. A nonlinear factor analysis of S\&P 500 index option returns. Journal of Finance, Vol. 61, Issue 5, 2006, p. 2325-2363.

[28] Lamsa V., Kullaa J. Nonlinear factor analysis in structural health monitoring to remove environmental effects. 6th International Workshop on Structural Health Monitoring, Stanford, CA, USA, 2007, p. 1092-1099.

[29] Douglas S. C. Detection of aliasing in image sequences using nonlinear factor analysis. Proceedings of the 10th International Conference on Latent Variable Analysis and Signal Separation, 2012, p. 486-493.

[30] Curran P. J. A moderated nonlinear factor model for the development of commensurate measures in integrative data analysis. Multivariate Behavioral Research, Vol. 49, Issue 3, 2014, p. 502-503.

[31] Honkela H. L. A. Bayesian nonlinear independent component analysis by multi-layer perceptrons. Advances in Independent Component Analysis, Springer, 2000, p. 93-121.

[32] Liu H. T., Chang C. Q., Luk K. D. K., Hu Y. Comparison of blind source separation methods in fast somatosensory-evoked potential detection. Journal of Clinical Neurophysiology. Vol. 28, Issue 2, 2011, p. 170-177.

[33] Farina D., Fevotte C., Doncarli C., Merletti R. Blind separation of linear instantaneous mixtures of nonstationary surface myoelectric signals. IEEE Transactions on Biomedical Engineering, Vol. 51, Issue 9, 2004, p. 1555-1567. 


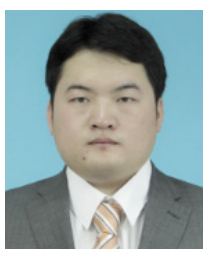

Wei Cheng received Ph.D. degree in Department of Mechanical Engineering from Xi'an Jiaotong University, Xi'an, China, in 2012. Now he works at Department of Mechanical Engineering of Xi'an Jiaotong University. His current research interests include blind source separation and evaluation.

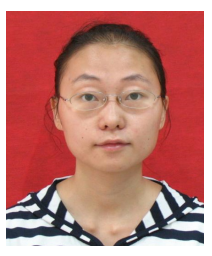

Lin Gao received Ph.D. degree in Department of Biomedical Engineering from Xi'an Jiaotong University, Xi'an, China, in 2013. Now she works at Department of Biomedical Engineering of Xi'an Jiaotong University. Her current research interests include biomedical signal processing.

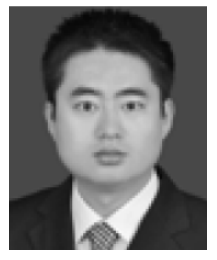

Jie Zhang received B.S. degree in mechanical engineering from Sichuan University, Chengdu, China, in 2009. He is currently pursuing Ph.D. degree in mechanical engineering of Xi'an Jiaotong University, Xi'an, China. His current research interests include vibration signal processing.

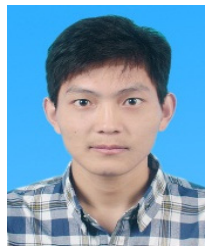

Jiantao Lu received Bachelor degree in School of Mechanical Engineering from Zhengzhou University, Zhengzhou, China, in 2012. Now as a Ph.D. student of Xi'an Jiaotong University, his current research interests include nonlinear blind source separation and fault diagnosis. 